\title{
The determinants of dominance relationships among subordinate females in the cooperatively breeding meerkat
}

\author{
N.K. Thavarajah ${ }^{\mathrm{a}, \mathrm{b}, *}$, M. Fenkes ${ }^{\mathrm{b}, \mathrm{c}}$ and T.H. Clutton-Brock ${ }^{\mathrm{a}, \mathrm{b}, \mathrm{d}}$ \\ ${ }^{a}$ Large Animal Research Group, Department of Zoology, University of Cambridge, \\ Downing Street, Cambridge CB2 3EJ, UK \\ ${ }^{\mathrm{b}}$ Kalahari Meerkat Project, Kuruman River Reserve, 8467 Van Zylsrus, South Africa \\ ${ }^{c}$ Animal Behaviour, Institute of Evolutionary Biology and Environmental Studies, \\ University of Zürich, Winterthurerstrasse, 190, 8057 Zürich, Switzerland \\ ${ }^{\mathrm{d}}$ Mammal Research Institute, Pretoria University, 0002 Pretoria, South Africa \\ *Corresponding author's e-mail address: nathanthavarajah@gmail.com
}

\begin{abstract}
In cooperatively breeding species with high reproductive skew, a single breeding female is dominant to all other group members, but it is not yet known if there are consistent dominance relationships among subordinates. In this study on meerkats (Suricata suricatta), we used naturally observed dominance assertions and submissive interactions within dyads of subordinate females to investigate: (i) whether or not a dominance structure exists among them and what factors influence dominance relationships; and (ii) how dominance may influence the future reproductive success of subordinate females. Our study indicates that superiority in age and weight provide a competitive advantage during conflicts among subordinate females and that females who consistently dominate in these contests are subsequently more likely to attain a dominant breeding position. This provides a starting point for further investigations into dominance structure among subordinates in meerkat societies and other cooperative breeders.
\end{abstract}

Keywords

individual attributes, social hierarchy, dyadic dominance relationships.

\section{Introduction}

Where animal societies exist, groups are often organised by dominance relationships among group members, creating a hierarchy (Apps, 1992; Pusey 
\& Packer, 1997). Within groups where single individuals are able to monopolise resources, the dominance structure may be described as despotic, with weak tolerance levels among group members and well-differentiated relationships organised in a strong linear hierarchy (Archie et al., 2006; Bergstrom \& Fedigan, 2013). This type of hierarchy has been described in cooperatively breeding invertebrate societies (Cronin \& Field, 2007), whereas empirical evidence of this kind is lacking in cooperatively breeding mammals, where one animal of each sex is often dominant over all others and monopolises reproduction. Where such societies occur, the mechanisms underlying social dominance exerted by dominant breeders towards non-breeding subordinates are relatively well known (Clutton-Brock, 2009). However, as interactions among subordinates are more subtle and rare, it is often unclear if dominance relationships among them do not exist, or if they are difficult to define through specific behaviours (de Luca \& Ginsberg, 2001). This is seen in African wild dogs, Lycaon pictus (Spiering et al., 2010), dwarf mongooses, Helogale parvula (Keane et al., 1994), Damaraland mole rats, Fukomys damarensis (Burland et al., 2004) and common marmosets, Callithrix jacchus (Saltzman et al., 2009).

Dominance has been defined as an attribute of repeated, agonistic interactions between two individuals with a consistent outcome in favour of the same dyad member, giving dominance a crucial organising function in a wide range of social contexts (Drews, 1993). Dominance relationships are essential in order to maintain group cohesion in animal societies (Apps, 1992) by reducing aggression and providing individuals with the knowledge of their place in the group (Savin-Williams, 1976). Dominance is also correlated with greater access to food sources and higher reproductive success of dominant individuals than subordinate ones in a variety of species (e.g., in red deer, Cervus elaphus: Clutton-Brock et al., 1986; hyenas, Crocuta crocuta: Frank, 1986; barnacle geese, Branta leucopsis: Stahl et al., 2001; chimpanzees, Pan troglodytes: Wittig \& Boesch, 2003 and meerkats: Clutton-Brock et al., 2006). As a result, the factors conferring dominance acquisition or acting as cues to aid in the resolution of conflicts are of considerable interest (Stanback, 1994). Previous work suggests that individual attributes such as age or size, which indicate an individual's resource holding potential (RHP) (Parker, 1974; Maynard Smith \& Parker, 1976), are fundamental in the organisation of dominance relationships and contests between individuals are often settled according to asymmetries in these attributes 
(Chase, 1974; Clutton-Brock et al., 1986; Beaugrand et al., 1991; Stanback, 1994; Côté, 2000; Stahl et al., 2001; Chase et al., 2002; Beacham, 2003; Wittig \& Boesch, 2003; Archie et al., 2006; Prenter et al., 2008). Asymmetries in attributes such as age need only be small for dominance relationships to form, as seen in its extreme in siblicidal species (e.g., the blue-footed booby, Sula nebouxii (Drummond \& Garcia Chavelas, 1989) and the black kite, Milvus migrans (Vinuela, 1999)), where individuals often hatch several days apart and the first born is likely to have a competitive advantage. Individual asymmetries are common in cooperatively breeding societies, where helpers are often retained offspring and may remain in their natal group for several generations (e.g., in dwarf mongooses (Keane at al., 1994), Damaraland mole-rats, Fukomys damarensis (Cooney \& Bennett, 2000), and African wild dogs (Spiering et al., 2010)). However, it remains largely unknown whether or not the asymmetries that exist may contribute to the formation of a dominance structure among the subordinate helpers and how individuals may benefit from dominating their subordinate counterparts.

Meerkats are members of the mongoose family, Herpestidae, and are found throughout the dry parts of Southern Africa (Clutton-Brock et al., 1998a). Meerkats live in stable groups of 2-50 individuals (Clutton-Brock et al., 2005) with despotic characteristics (Kutsukake \& Clutton-Brock, 2008), commonly consisting of a dominant pair who are the main breeders and an approximately equal number of subordinates of both sexes that are often retained offspring and contribute towards cooperative activities (Clutton-Brock et al., 2002). There are high levels of reproductive skew, as the dominant female monopolises breeding (Clutton-Brock et al., 2001) through a combination of aggression through dominance assertion and eviction (Clutton-Brock et al., 1998b; Young et al., 2006). Consequently, attributes that increase competitive ability exert a strong influence on female behaviour (Clutton-Brock et al., 2006). Dominant females exhibit a secondary growth phase following dominance acquisition (Russell et al., 2004), suggesting size as a beneficial attribute in maintaining dominance in this species. In addition, when subordinate females compete for a vacant dominant breeding position, individual attributes determine who is likely to succeed, suggesting larger size and older age are advantageous in the acquisition of a dominant breeding position (Clutton-Brock et al., 2006; Hodge et al., 2008). It remains largely unknown if and how a dominance structure may be established among subordinate female meerkats while they are still under the despotic rule of a 
dominant breeding female and how this might influence their future reproductive success. The existence of a dominance structure among subordinate females and the factors that influence its organization could hold profound implications for the outcome of subsequent rank-related conflicts (i.e., when a breeding position becomes vacant).

This study investigates whether or not there are consistent dominance relationships among subordinate female meerkats. By considering dominance assertions and submissive interactions between subordinate females, we investigated the following questions: i) Is the outcome of dominance interactions between subordinate females determined by attributes such as age and weight? and ii) Are subordinate females that consistently exert dominance over other subordinate females more likely to subsequently acquire a dominant breeding role?

\section{Material and methods}

\subsection{Study animals and field site}

We collected data at the Kalahari Meerkat Project (KMP), Kuruman River Reserve, close to Vanzylsrus $\left(26^{\circ} 58^{\prime} \mathrm{S}, 21^{\circ} 49^{\prime} \mathrm{E}\right)$ in the Southern Kalahari, South Africa (see Russell et al., 2002 for details on the climate of this region). This population of meerkats is habituated to close observation $(<2 \mathrm{~m})$ and has been studied since, 1993 as part of a long term research project. To aid in long term identification, all animals were fitted with an Identipet ${ }^{\circledR}$ (Johannesburg, South Africa) microchip transponder during the month following their birth, or upon immigration into the study population. We further identified individuals based on patterns of hair dye marks on the fur, which were maintained regularly by observers while animals rested at the sleeping burrow. Age and/or date of birth were known for almost all individuals, except for meerkats that had immigrated from outside of the study population. We only included individuals with a known date of birth in our analyses.

\subsection{Data collection and analysis}

Over 12 years of data (between January, 2000 and March, 2012) analysed in this study were derived from the KMP's comprehensive long-term database. Data were collected by a succession of trained research assistants who observed 49 meerkat groups 2-4 times per week. They collected demographic, life history, and up to four hours of behavioural data per observation day. 
Behavioural data collection followed the ad libitum sampling rule (Martin \& Bateson, 2007), using a detailed, pre-defined catalogue of relevant behaviours (Kalahari Meerkat Project Protocol, 1996 to 2010). The ad libitum method is biased towards recording individuals and behaviours that are particularly conspicuous and it does not provide a complete representation of the events occurring in the whole group. However, it does allow the recording of rare but important events (Martin \& Bateson, 2007). Research assistants also weighed all individuals present per group during each observation period by enticing them onto a set of laboratory scales using a minimal amount of water and hard-boiled egg.

In this study we described and analysed dominance relationships among subordinate females using observed dominance assertions and submissive interactions. Dominance assertion behaviours include glaring (the dominant individual provocatively staring at the asserted individual), chin-rubbing (the dominant individual rubbing its chin against the face/neck region of the asserted individual), hip-slamming (the dominant individual slamming its hindquarters against the asserted individual), and charging/attacking as the most extreme forms of dominance assertion. Submissive behaviours include peeping (a high frequent, high pitched call made by the submissive individual upon contact or near-contact with the other individual), and grovelling (the submissive individual crouching on its forelegs, the head held low above the ground and stretched towards the other individual, this posture almost always accompanied by peeping) (see also Kutsukake \& Clutton-Brock, 2006, 2008, for detailed definitions of dominance assertions and submissive interactions). Due to our sampling method and the limitations of our longitudinal behavioural data set, we were able to determine most, but not all dominance relationships among subordinate females in the study population. We recorded a total of 1209 dyadic dominance interactions using assertion behaviours where at least one dominance assertion occurred within a dyad, 1012 of which were between females with an age difference (i.e., birth date $\geqslant 1$ day apart) and, 197 between same-aged females (i.e., born on the same day). We observed a total of 1214 dyadic dominance interactions using submissive behaviours where at least one submission occurred within a dyad, 1112 of which were between differently-aged females and 102 between same-aged females. We chose to condense each dyadic dominance relationship into one data point, conveying the number of dominance assertions and submissions of each partner towards the other. We pooled 
dominance assertions and submissive interactions per dyad and scored the number of times each female dominated her partner, either through asserting dominance or receiving submission. This cumulation of data enabled us to analyse the determinants of dominance within dyads, as opposed to using individual performance during single dominance interactions in relation to the rest of the group as the observational unit of analysis.

\subsection{Statistical analyses}

All statistics were conducted using R (R Development Core Team, 2009). Because we investigated dyadic relationships between females that interacted frequently and the resulting count data were thus likely to be dependent, we used non-parametric methods for statistical analyses.

In order to reduce the data set to non-ambiguous events, we compared the dominance score of both females in each dyad using binomial tests and excluded those lacking a significant difference (regarded as ties) from our following analyses. The conflicts in the remaining dyads ended in consistent favour of one female over the other (Drews, 1993), which we imply when using the term 'dominance relationship' in the following text (see also Pusey $\&$ Packer, 1997). The remaining total number of dyads entering further analyses was 157, 141 of which consisted of differently aged females, and 16 of same-aged females. A total of 170 females were involved in these dominance relationships.

\subsubsection{Influence of individual attributes on dominance relationships}

For dominance interactions between differently-aged females, we assessed if the outcome was affected by age difference, weight difference, or both. We calculated the average weight of each female seven days prior to/post each dominance interaction. We then categorized the weight of each female as either 'heavier' $(\mathrm{H})$ or 'lighter' (L) within a dyad using the average of these weights per female. For dominance interactions between differentlyaged females, we then categorized each female per dyad as 'older' (O) and 'younger' $(\mathrm{Y})$ and counted the number of dyads in which the older or younger female dominated her partner. We compared the number of dyads in which each age-weight combination (older/heavier, $\mathrm{O} / \mathrm{H}$; older/lighter, $\mathrm{O} / \mathrm{L}$; younger/heavier, $\mathrm{Y} / \mathrm{H}$; younger/lighter, Y/L) was found for the consistently dominating female by means of a Pearson's chi-squared test.

For dominance interactions between same-aged females, we counted the number of dyads in which the heavier or the lighter female dominated over her partner and compared these counts using binomial tests. 
We noted the exact age difference in days (ranging between 0 and 1280 days and grouped into 4 arbitrary categories, i.e., $\leqslant 360$ days, $361-720$ days, 721-1080 days, 1081-1280 days) and weight differences in grams (ranging between 1.1 and 365.2 grams and grouped into 8 arbitrary categories, i.e., $\leqslant 50 \mathrm{~g}, 51-100 \mathrm{~g}, 101-150 \mathrm{~g}, 151-200 \mathrm{~g}, 201-250 \mathrm{~g}, 251-300 \mathrm{~g}, 301-350 \mathrm{~g}$, 351-365.2 g) between all females that interacted in dominance relationships in order to investigate if dominance relationships are more likely to occur where individual asymmetries are small rather than large.

\subsubsection{Acquisition of dominant breeding position}

We investigated whether or not the outcome of dominance relationships between subordinate females influences their likelihood of subsequently attaining a dominant breeding position. We assessed this using proportion tests with continuity correction on the number of subordinate females (pooling from dyads with an age difference and from same-aged dyads) that attained a dominant breeding position after they either (i) always dominated their partner(s) in dominance relationships or (ii) were always submissive to their partner(s). To avoid pseudo replication, each female entered the analysis only once, either as submissive or as dominant over all the females it interacted with. Females with ambiguous outcomes were thus excluded ( $N=34$ out of 170).

\section{Results}

\subsection{Influence of individual attributes on dominance relationships}

In 141 dyads of differently-aged subordinate females the outcome of dominance interactions was influenced by age and weight. Older/heavier females $(N=117)$ dominated others more often than older/lighter $(N=11)$, younger/heavier $(N=5)$, and younger/lighter females $(N=8)$. Older/ heavier females were dominant more often than expected by chance. (Pearson's chi-squared test: $\chi_{1}^{2}=24.014, p<0.0001$ ).

Dominance relationships between same-aged individuals occurred in 16 dyads, where heavier individuals $(N=13)$ were more likely to dominate their partners than lighter ones $(N=3)$ (Binomial test: $N=16, p=0.021)$.

We found that in most dyads (68.8\% of all 157 dyads) in which we observed dominance relationships, the age difference between females was less than 360 days, whereas higher age differences occurred much less frequently (Figure 1a). Weight differences within dyads were also primarily 

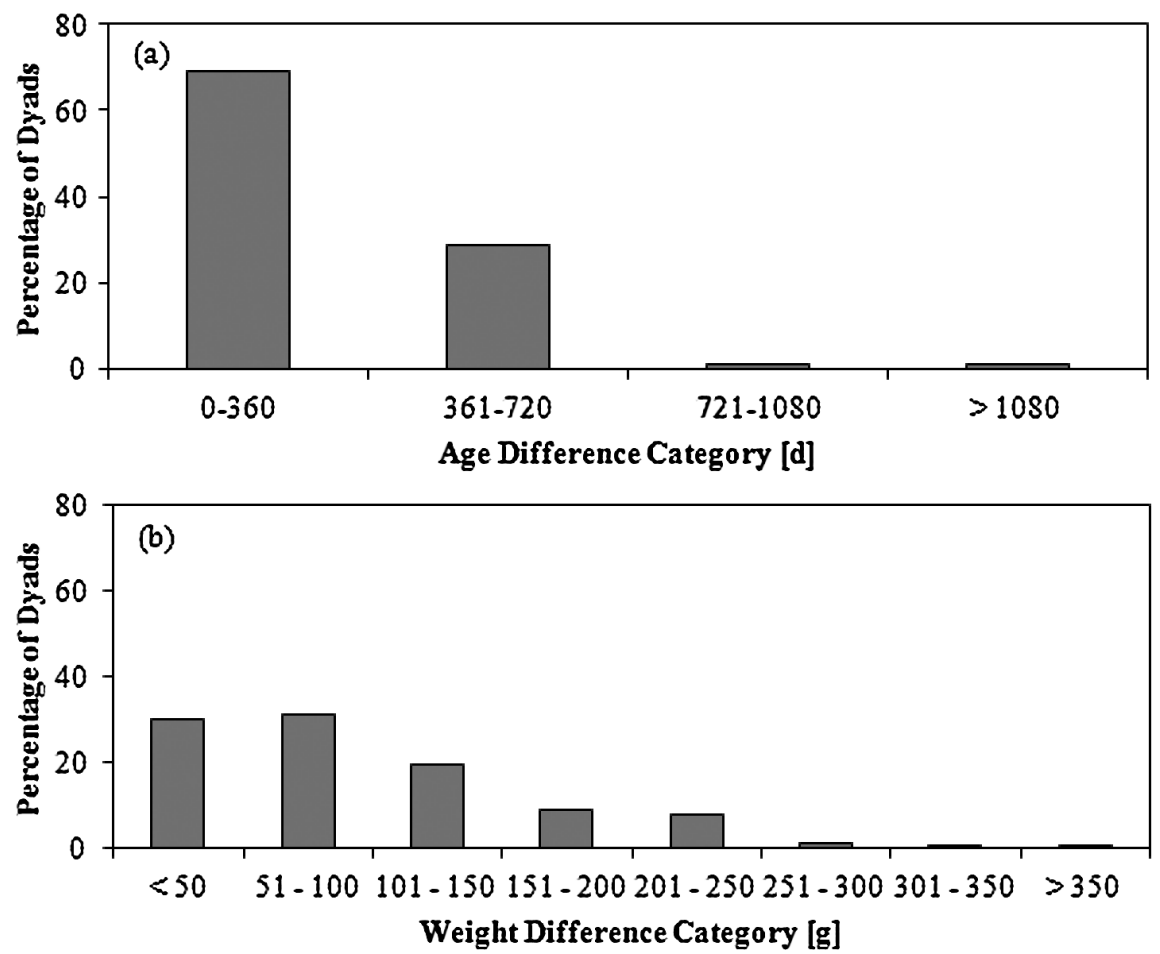

Figure 1. Percentage of dyads within which dominance relationships were formed, as categorised by (a) the age difference and (b) weight difference between dyadic partners. Dominance relationships were defined as relationships where one partner significantly more often dominated her dyadic partner, as assessed through binomial tests.

small ( $\leqslant 50 \mathrm{~g}$ in $29.9 \%$ of dyads and $51-100 \mathrm{~g}$ in $31.2 \%$ of dyads), whereas larger asymmetries in weight were found much less frequently (Figure 1b).

\subsection{Acquisition of dominant breeding position}

Out of 136 females, 50 always dominated their partners, whereas 86 were always submissive to their partners. A larger proportion of the dominating females than of the submissive ones subsequently attained a dominant breeding position (Proportion test with continuity correction: $N_{\text {dominant }}=26$ out of $50, N_{\text {submissive }}=13$ out of $\left.86, \chi_{1}^{2}=19.265, p<0.0001\right)$.

\section{Discussion}

Our results showed that some subordinate females establish dominance relationships with one another in meerkat societies. The structure of these 
relationships was non-random and was correlated with asymmetries in age and weight between females. In addition, the establishment of dominance relationships among subordinates may affect their future reproductive success, as females who dominated in these relationships increased their chances of subsequently attaining a dominant breeding position.

In meerkat groups, competition among counterparts is likely to have important individual consequences from birth through to maturation. Where multiple litters are born within the same babysitting burrow by multiple females, first born litters, being older and larger, possess a competitive advantage over subsequent litters (Clutton-Brock et al., 1998b), which may be born several days apart. This becomes evident in the greater survival rates of first born litters to emergence (Clutton-Brock et al., 2001). Age and weight have also been shown to be important factors influencing later life history events. They determine which individuals are more likely to succeed in attaining a vacant dominant breeding position (Clutton-Brock et al., 2006; Hodge et al., 2008), as well as having a positive influence on the length of dominant breeding tenure, reproductive success and offspring weight (Russell et al., 2004; Hodge et al., 2008).

Research on meerkats to date has largely overlooked the competitive relationships which may form among subordinates in the time period following independence ( $>3$ months of age) and until dispersal or the acquisition of a dominant breeding position. Although subordinate female meerkats often remain in their natal group as non-breeders for several years (Clutton-Brock et al., 1998b; Clutton-Brock, 2009), dominant breeder turnover is often slow and may in many cases exceed a subordinate's lifespan or membership in a group (Sharp \& Clutton-Brock, 2010). This aggravates the determination of possible benefits that subordinate females may gain from competing for dominance among their counterparts. We found a correlation between age and weight of subordinate females and the outcome of dominance relationships that are formed among them, with conflicts ending in consistent favour of older and heavier females. When the age of contestants was equal, the outcome of dominance relationships was influenced by weight, with conflicts ending in consistent favour of heavier females. Age and weight are not mutually exclusive variables and are likely to be correlated, however our results suggest that superiority in these attributes provide a competitive advantage during contests among subordinate females in meerkat societies. Due to the limitations of our data set regarding possible unknown dominance 
relationships, we did not produce a rank order of individuals pertaining to their dominance status within their group as a whole.

It is currently unknown whether or not dominance relationships among subordinate female meerkats may form in the absence of any immediate direct fitness or survival benefits (Clutton-Brock et al., 2001; Clutton-Brock et al., 2006), or if individuals form a queuing system which may have future benefits as shown in some social insect species (Cant et al., 2006; Cronin \& Field, 2007). In the cooperatively breeding hairy-faced hover wasp (Liostenogaster flavolineata) dominance relationship formation among subordinate females is age related and top-down aggression among subordinates serves to strengthen an age-based inheritance queue (Cronin \& Field, 2007). Cant et al. (2006) suggest that the frequency of aggression among subordinate individuals in group living species, in which a single dominant individual monopolises breeding, should be greatest nearer the front of an inheritance queue. Our observation that dyadic dominance relationships occur more often between subordinate female meerkats where individual asymmetries are relatively small, suggests a necessity for future research on this topic in order to better understand the causal factors influencing dominance relationship formation. In meerkat societies, contests may only occur between individuals who are closely matched in their competitive abilities, which has been observed in elephants Loxodonta africana (Archie et al., 2006). They may avoid contests with individuals who exhibit a clear difference in relative RHP due to the inherent physiological costs of dominance behaviour, i.e., rise in cortisol levels (Creel, 2001, 2005; Carlson et al., 2004). It has previously been shown that meerkats preferably disperse with similar-aged dispersal partners (Sharpe, 2005) and following subsequent new group formation, one female will become dominant while the others face aggression and once again eviction (Clutton-Brock, 2009). We suggest that similar-aged subordinate females may establish dominance relationships among their subordinate counterparts prior to dispersal and reproductive competition events in order to improve their prospects in later contests.

The benefits of forming dominance hierarchies for non-breeding subordinates in group living species have rarely been observed in nature, with evidence being largely confined to studies on social insects and some bird species (e.g., striped-backed wrens, Campylorhynchus nuchalis: Wiley \& Rabenold, 1984; ants, Dinoponera quadriceps: Monnin \& Peeters, 1999; 
hairy-faced hover wasps: Cronin \& Field, 2007). By securing a high ranking position among subordinate counterparts, individuals can increase their chances of subsequently securing a dominant breeding position by either outliving or eventually outcompeting and supplanting the existing dominant breeder (Cant et al., 2006). As with many cooperative breeding societies, meerkat societies exhibit high levels of reproductive skew (Clutton-Brock et al., 2001), making it favourable for individuals to attain a dominant breeding position. We found that subordinate females who always dominated their partners in dyadic dominance relationships were subsequently more likely to attain a dominant breeding position than females who never dominated other subordinate females. This result suggests that the dominance relationships which are formed among subordinate female meerkats may be a behavioural strategy demonstrated to secure a high ranking position and therefore greater chances of future reproductive success.

In conclusion, this study represents the first preliminary evidence and description of dominance relationships among subordinate females in a cooperatively breeding mammal with high reproductive skew. We have shown that subordinate female meerkats form dominance relationships and that age and weight influence the direction of these relationships. In addition, subordinate females who dominated their partner in dyadic dominance relationships were more likely to later attain a dominant breeding position, over those who were submissive. These results suggest that a more complex dominance hierarchy may exist within meerkat societies than has been previously described and that subordinate females may gain future benefits from forming dominance relationships. Future research should look to further deepen our knowledge of the dominance relationships among non-breeding subordinates in order to gain a better understanding of the social dynamics and reproductive strategies in cooperatively breeding species.

\section{Acknowledgements}

We thank the Kotze family and Northern Cape Conservation for allowing us to conduct research in the Kalahari; all previous project management and the many research volunteers, field staff, PhD students, MSc students and postdoctorates that have assisted with data collection since the project began. We are grateful to Tom Flower and Marta Manser for their invaluable comments on earlier drafts of this paper and to Dave Gaynor for statistics advice and 
discussion on data analyses; we are also grateful to three anonymous reviewers for very detailed comments and further improvements.

\section{References}

Apps, P. (1992). Wild ways, 4th edn. - Southern Book Publishers, Halfway House.

Archie, E.A., Morrison, T.A., Foley, C.A.H., Moss, C.J. \& Alberts, S.C. (2006). Dominance rank relationships among wild female African elephants, Loxodonta africana. - Anim. Behav. 71: 117-127.

Beacham, J.L. (2003). Models of dominance hierarchy formation: effects of prior experience and intrinsic traits. - Behaviour 140: 1275-1303.

Beaugrand, J.P., Goulet, C. \& Payette, D. (1991). Outcome of dyadic conflict in male green swordtail fish, Xiphophorus helleri: effects of body size and prior dominance. - Anim. Behav. 41: 417-424.

Bergstrom, M.L. \& Fedigan, L.M. (2013). Dominance style of female white-faced capuchins. — Am. J. Phys. Anthropol. 150: 591-601.

Burland, T.M., Bennett, N.C., Jarvis, J.U.M. \& Faulkes, C.G. (2004). Colony structure and parentage in wild colonies of co-operatively breeding Damaraland mole-rats suggest incest avoidance alone may not maintain reproductive skew. - Mol. Ecol. 13: 2371-2379.

Cant, M.A., Llop, J.B. \& Field, J. (2006). Individual variation in social aggression and the probability of inheritance: theory and field test. - Am. Nat. 167: 837-852.

Carlson, A.A., Young, A.J., Russell, A.F., Bennett, N.C., McNeilly, A.S. \& Clutton-Brock, T.H. (2004). Hormonal correlates of dominance in meerkats (Suricata suricatta). Horm. Behav. 46: 141-150.

Chase, I.D. (1974). Models of hierarchy formation in animal societies. - Behav. Sci. 19: 374-382.

Chase, I.D., Tovey, C., Spangler-Martin, D. \& Manfredonla, M. (2002). Individual differences versus social dynamics in the formation of animal dominance hierarchies. - Proc. Natl. Acad. Sci. USA 99: 5744-5749.

Clutton-Brock, T.H. (2009). Structure and function in mammalian societies. - Phil. Trans. Roy. Soc. B. 364: 3229-3242.

Clutton-Brock, T.H., Albon, S.D. \& Guinness, F.E. (1986). Great expectations: dominance, breeding success and offspring ratios in red deer. - Anim. Behav. 34: 460-471.

Clutton-Brock, T.H., Brotherton, P.N.M., Smith, R., McIlrath, G.M., Kansky, R., Gaynor, D., O’Riain, M.J. \& Skinner, J.D. (1998a). Infanticide and expulsion of females in a cooperative mammal. — Proc. Roy. Soc. Lond. B: Biol. Sci. 265: 2291-2295.

Clutton-Brock, T.H., Gaynor, D., Kansky, R., MacColl, A.D.C., McIlrath, G., Chadwick, P., Brotherton, P.N.M., O’Riain, J.M., Manser, M. \& Skinner, J.D. (1998b). Costs of cooperative behaviour in suricates (Suricata suricatta). — Proc. Roy. Soc. Lond. B: Biol. Sci. 265: 185-190.

Clutton-Brock, T.H., Brotherton, P.N.M., Russell, A.F., O’Riain, M.J., Gaynor, D., Kansky, R., Griffen, A., Manser, M., Sharpe, L., McIlrath, G.M., Small, T., Moss, A. \& Monfort, S. (2001). Cooperation, control and concession in meerkat groups. — Science 291: 478-480. 
Clutton-Brock, T.H., Russell, A.F., Sharpe, L.L., Young, A.J., Balmforth, Z. \& McIlrath, G.M. (2002). Evolution and development of sex differences in cooperative behaviour in meerkats. - Science 297: 253-256.

Clutton-Brock, T.H., Russell, A.F., Sharpe, L.L. \& Jordan, N.R. (2005). 'False feeding' and aggression in meerkat societies. - Anim. Behav. 69: 1273-1284.

Clutton-Brock, T.H., Hodge, S.J., Spong, G., Russell, A.F., Jordan, N.R., Bennett, N.C., Sharpe, L.L. \& Manser, M.B. (2006). Intrasexual competition and sexual selection in cooperative mammals. - Nature 444: 1065-1068.

Cooney, R. \& Bennett, N.C. (2000). Inbreeding avoidance and reproductive skew in a cooperative mammal. — Proc. Roy. Soc. Lond. B: Biol. Sci. 267: 801-806.

Côté, S.D. (2000). Dominance hierarchies in female mountain goats: stability, aggressiveness and determinants of rank. - Behaviour 37: 1541-1566.

Creel, S. (2001). Social dominance and stress hormones. — Trends Ecol. Evol. 16: 491-497.

Creel, S. (2005). Dominance, aggression, and glucocorticoid levels in social carnivores. J. Mammal. 86: 255-264.

Cronin, A.L. \& Field, J.P. (2007). Rank and colony defence against conspecifics in a facultatively eusocial hover wasp. - Behav. Ecol. 18: 331-336.

de Luca, D.W. \& Ginsberg, J.R. (2001). Dominance, reproduction and survival in banded mongooses: towards an egalitarian social system? — Anim. Behav. 61: 17-30.

Drews, C. (1993). The concept and definition of dominance in animal behaviour. - Behaviour 125: 283-313.

Drummond, H. \& Garcia Chavales, C. (1989). Food shortage influences sibling aggression in the blue-footed booby. - Anim. Behav. 37: 806-819.

Frank, L.G. (1986). Social organization of the spotted hyaena Crocuta crocuta. II. Dominance and reproduction. - Anim. Behav. 34: 1510-1527.

Hodge, S.J., Manica, A., Flower, T.P. \& Clutton-Brock, T.H. (2008). Determinants of reproductive success in dominant female meerkats. - J. Anim. Ecol. 77: 92-102.

Keane, B., Waser, P.M., Creel, S.R., Creel, N.M., Elliott, L.F. \& Minchella, D.J. (1994). Subordinate reproduction in dwarf mongooses. - Anim. Behav. 47: 65-75.

Kutsukake, N. \& Clutton-Brock, T.H. (2006). Social functions of allogrooming in cooperatively breeding meerkats. - Anim. Behav. 72: 1059-1068.

Kutsukake, N. \& Clutton-Brock, T.H. (2008). Do meerkats engage in conflict management following aggression? Reconciliation, submission and avoidance. - Anim. Behav. 75: 1441-1453.

Martin, P. \& Bateson, P. (2007). Measuring behaviour, 3rd edn. - Cambridge University Press, Cambridge.

Maynard Smith, J. \& Parker, G.H. (1976). The logic of asymmetric contests. - Anim. Behav. 24: 159-175.

Monnin, T. \& Peeters, C. (1999). Dominance hierarchy and reproductive conflicts among subordinates in a monogynous queenless ant. - Behav. Ecol. 10: 323-332.

Parker, G.A. (1974). Assessment strategy and the evolution of animal conflicts. - J. Theor. Biol. 47: 223-243. 
Prenter, J., Taylor, P.W. \& Elwood, R.W. (2008). Large body size for winning and large swords for winning quickly in swordtail males, Iphophorus helleri. - Anim. Behav. 75: 1981-1987.

Pusey, A.E. \& Packer, C. (1997). The ecology of relationships. — In: Behavioural ecology: an evolutionary approach, 4th edn. (Krebs, J.R. \& Davies, N.B., eds). Blackwell, Oxford, p. 254-287.

Russell, A.F., Carlson, A.A., McIlrath, G.M., Jordan, N.R. \& Clutton-Brock, T.H. (2004). Adaptive size modification by dominant female meerkats. — Evolution 58: 1600-1607.

Russell, A.F., Clutton-Brock, T.H., Brotherton, P.N., Sharpe, L.L., McIlrath, G.M., Dalerum, F.D., Cameron, E.Z. \& Barnard, J.A. (2002). Factors affecting pup growth and survival in cooperatively breeding meerkats Suricata suricatta. — J. Anim. Ecol. 71: 700-709.

Saltzman, W., Schultz-Darken, N.J. \& Abbott, D.H. (1996). Behavioural and endocrine predictors of dominance and tolerance in female marmosets, Callithrix jacchus. - Anim. Behav. 51: 657-674.

Savin-Williams, R.C. (1976). An ethological study of dominance formation and maintenance in a group of human adolescents. — Child Dev. 47: 972-979.

Sharp, S.P. \& Clutton-Brock, T.H. (2010). Reproductive senescence in a cooperatively breeding mammal. — J. Anim. Ecol. 79: 176-183.

Sharpe, L.L. (2005). Frequency of social play does not affect dispersal partnerships in wild meerkats. - Anim. Behav. 70: 559-569.

Spiering, P.A., Somers, M.J., Maldonado, J.E., Wildt, W.E. \& Gunther, M.S. (2010). Reproductive sharing and proximate factors mediating cooperative breeding in the African wild dog (Lycaon pictus). — Behav. Ecol. Sociobiol. 64: 583-592.

Stahl, J., Tolsma, P.H., Loonen, M.J. \& Drent, R.H. (2001). Subordinates explore but dominants profit: resource competition in high Arctic barnacle goose flocks. - Anim. Behav. 61: 257-264.

Stanback, M.T. (1994). Dominance within broods of the cooperatively breeding acorn woodpecker. - Anim. Behav. 47: 1121-1126.

Vinuela, J. (1999). Sibling aggression, hatching asynchrony, and nestling mortality in the black kite (Milvus migrans). — Behav. Ecol. Sociobiol. 45: 33-45.

Wiley, R.H. \& Rabenald, K.N. (1984). The evolution of cooperative breeding by delayed reciprocity and queuing for favourable social positions. - Evolution 38: 609-621.

Wittig, R.M. \& Boesch, C. (2003). Food competition and linear dominance hierarchy among female chimpanzees of the Taï National Park. — Int. J. Primatol. 24: 847-867.

Young, A.J., Carlson, A.A., Monfort, S.L., Russell, A.F., Bennett, N.C. \& Clutton-Brock, T.H. (2006). Stress and suppression of subordinate reproduction in cooperatively breeding meerkats. — Proc. Natl. Acad. Sci. USA 103: 12005-12010. 